\title{
CNO behaviour in planet-harbouring stars
}

\section{Nitrogen abundances in stars with planets ${ }^{\star}$}

\author{
L. Suárez-Andrés ${ }^{1,2}$, G. Israelian ${ }^{1,2}$, J. I. González Hernández ${ }^{1,2}$, V. Zh. Adibekyan ${ }^{3}$, E. Delgado Mena ${ }^{3}$, \\ N. C. Santos ${ }^{3,4}$, and S. G. Sousa ${ }^{3,4}$ \\ 1 Instituto de Astrofísica de Canarias, 38205 La Laguna, Tenerife, Spain \\ e-mail: 1suarez@iac.es \\ 2 Depto. Astrofísica, Universidad de La Laguna (ULL), 38206 La Laguna, Tenerife, Spain \\ 3 Instituto de Astrofísica e Ciências do Espaço, Universidade do Porto, CAUP, Rua das Estrelas, 4150-762 Porto, Portugal \\ ${ }^{4}$ Departamento de Física e Astronomia, Faculdade de Cîencias, Universidade do Porto, 4169-007 Porto, Portugal
}

Received 7 March 2016 / Accepted 23 April 2016

\begin{abstract}
Context. Carbon, nitrogen, and oxygen (CNO) are key elements in stellar formation and evolution, and their abundances should also have a significant impact on planetary formation and evolution.

Aims. We present a detailed spectroscopic analysis of 74 solar-type stars, 42 of which are known to harbour planets. We determine the nitrogen abundances of these stars and investigate a possible connection between $\mathrm{N}$ and the presence of planetary companions. Methods. We used VLT/UVES to obtain high-resolution near-UV spectra of our targets. Spectral synthesis of the NH band at $3360 \AA$ was performed with the spectral synthesis codes MOOG and FITTING.

Results. We identify several spectral windows from which accurate $\mathrm{N}$ abundance can be obtained. Nitrogen distributions for stars with and without planets show that planet hosts are nitrogen-rich when compared to single stars. However, given the linear trend between $[\mathrm{N} / \mathrm{Fe}]$ vs. $[\mathrm{Fe} / \mathrm{H}]$, this fact can be explained as being due to the metal-rich nature of planet hosts.

Conclusions. We conclude that reliable $\mathrm{N}$ abundances can be derived for metal-rich solar type stars from the near UV molecular band at $3360 \AA$. We confirm a linear trend between $[\mathrm{N} / \mathrm{Fe}]$ and metallicity expected from standard models of Galactic chemical evolution.
\end{abstract}

Key words. stars: abundances - stars: chemically peculiar - planetary systems

\section{Introduction}

The study of $\mathrm{C}, \mathrm{N}$, and $\mathrm{O}$ in stars is crucial because they are the most abundant elements after $\mathrm{H}$ and $\mathrm{He}$. These elements play an important role in stellar interiors because they generate energy through the CNO cycle, thereby affecting the lifetime of the stars (Liang et al. 2001)

Nitrogen is created in a different nucleosynthetic process from that giving rise to carbon and oxygen. Whereas for carbon and oxygen the dominant production modes are the $\alpha$-chain reactions, for nitrogen the dominant production mode lies in the rearrangement of nuclei during the CNO cycle (see Maeder 2009). One important question regarding $\mathrm{N}$ is its origin. Since $\mathrm{N}$ needs $\mathrm{C}$ and $\mathrm{O}$ to be formed, if it is formed from pre-existing $\mathrm{C}$ and $\mathrm{O}$ in the star, it is called "secondary". If, instead, the carbon and oxygen are produced in the star itself and then used to produce nitrogen, then the nitrogen is called "primary". Several studies have proved that production of nitrogen at low metallicities comes from primary rather than secondary sources (Pagel \& Edmunds 1981; Bessell \& Norris 1982; Carbon et al. 1987; Henry et al. 2000; Israelian et al. 2004). At higher metallicities, secondary processes dominate.

* Based on observations collected with the UVES spectrograph at the 8-m Very Large Telescope (VLT) - program IDs: 074.C-0134(A), 075.D-0453(A), 086.D-0082(A), 093.D-0328(A), installed at the Cerro Paranal Observatory.
Two main sources for primary production have been proposed:

1. Rotating massive stars, implying detached $\mathrm{N}$ and $\mathrm{Fe}$ abundances and overabundance of $\mathrm{N}$ regarding $\mathrm{Fe}$ (Maeder \& Meynet 2000).

2. Intermediate-mass stars $\left(4-8 M_{\odot}\right)$ during their thermally pulsing AGB phase through $\mathrm{CNO}$ processing in the convective envelope (Marigo 2001; van den Hoek \& Groenewegen 1997). The contribution by massive stars is negligible (Liang et al. 2001; Pettini et al. 2002), so dominant contributors are intermediate- and low-mass stars (ILMS). These ILMSs are also a source for secondary production.

Gonzalez (1997) and Santos et al. (2001) discovered that, on average, planet hosts are more metal-rich than "single" stars (stars with no known companion planet; from now on designated as single stars). Two hypotheses have been suggested to explain this anomaly:

1. Self enrichment: this scenario should be triggered by the action of hot jupiters migrating from outside the protoplanetary disc. During this migration, planetesimals from the disc are accreted on to the star. This mechanism would be efficient only during the first 20-30 Myr or later when the surface convection layers of the star for the first time attains its minimum size configuration (Chavero et al. 2010). Israelian et al. (2001, 2003) found evidence of pollution, 
suggesting the infall of a planet, in HD 82943 (see, however, Mandell et al. 2004; Ghezzi et al. 2009).

The self-enrichment scenario could lead to a relative overabundance of refractories, such as $\mathrm{Si}, \mathrm{Mg}, \mathrm{Ca}, \mathrm{Ti}$, and the iron-group elements, compared to volatiles, such as $\mathrm{C}, \mathrm{N}$, $\mathrm{O}, \mathrm{S}$, and $\mathrm{Zn}$. The accretion of small planets may affect the composition of the convective layer of the stars (see Théado \& Vauclair 2012, and references therein). To how great an extent this affects the accretion of volatiles such as C, N, and O (see González Hernández et al. 2013) remains unanswered.

2. Primordial cloud: Santos et al. (2001, 2002, 2003) proposed that this over-abundance most probably is caused by a metalrich primordial cloud. They claimed that the observed abundances are representative of the primordial cloud where the star was formed. This idea is supported by models of planet formation and evolution based on the core-accretion process (e.g. Ida \& Lin 2004; Mordasini et al. 2012)

Later abundance studies on stars with and without planets confirmed the primordial cloud as the most likely reason for the metal-rich nature of planet-host stars (Santos et al. 2004, 2005; Valenti \& Fischer 2005), although this correlation is valid for giant planet hosts only (Sousa et al. 2008, 2011b; Buchhave et al. 2012)

Interestingly, recent studies suggest that specific element abundances may have a particularly relevant role in the planet formation process (Adibekyan et al. 2014, 2015) or in its composition (Santos et al. 2015). The abundances of volatiles (such as $\mathrm{C}, \mathrm{N}$, and $\mathrm{O}$ ) may be particularly relevant in this respect. This consideration prompted the study of specific chemical abundances in the planet hosts. There are very few studies of nitrogen in solar-type stars owing to the lack of strong atomic lines. Ecuvillon et al. (2004) studied the abundance of nitrogen of 91 solar type stars. Using both the $\mathrm{NH}$ molecular band at $3360 \AA$ and the NI atomic line at $7468 \AA$, they rejected the self-enrichment scenario as a formation source on the grounds that they could find no underabundances of volatiles compared to refractory elements. They showed that the $[\mathrm{N} / \mathrm{H}]$ abundance scales perfectly with metallicity for both planet-host and comparison samples. They also found no difference for nitrogen in the $[\mathrm{N} / \mathrm{Fe}]$ abundance ratios when comparing stars with and without planets.

More recently, Da Silva et al. (2015), using the $\mathrm{CN}$ band at $4215 \AA$, have studied the abundance of nitrogen in 140 dwarf stars. They found a steeper slope for the $[\mathrm{N} / \mathrm{Fe}]$ versus $[\mathrm{Fe} / \mathrm{H}]$ abundance ratios than Ecuvillon et al. (2004).

The main problem in studying nitrogen abundances is the lack of strong nitrogen lines in the red part of the spectrum so that near-UV measurements are required. We are forced to study the very crowded molecular band at $3360 \AA$, where continuum determination is not straightforward.

The purpose of this study is to extend the sample of Ecuvillon et al. (2004) and investigate in detail nitrogen abundances using the $3360 \AA \mathrm{NH}$ molecular band. We have performed a systematic study of nitrogen abundances in dwarf stars with a wide range of stellar parameters. The strategy and methodology followed in this study is the same as that stated in Ecuvillon et al. (2004), but with a larger sample and higherquality spectra.

We have also determined the kinematic properties of our sample. Then using the kinematic and chemical properties of the stars, we separated them into different stellar populations to investigate the $\mathrm{N}$ abundances within a Galactic context.
Table 1. Observing logs for the campaigns.

\begin{tabular}{lc}
\hline \hline Campaign & Observing dates \\
\hline 074.C-0134(A) & 21-22 Dec. 2004 \\
075.D-0453(A) & First semester 2005 \\
086.D-0082(A) & Oct. 2010-Nov. 2011 \\
093.D-0328(A) & Mar.-Jul. 2014 \\
\hline
\end{tabular}

This work is the first step in comparing CNO abundances of planet-host stars with the $\mathrm{CNO}$ abundances of their planets (through transmission spectroscopy or direct spectroscopy, as in the case of Spitzer). Testing and improving planetary formation models will play a key role in future studies of habitability, CNO being key elements for life.

\section{Sample description}

The high-resolution spectra analysed in this study were obtained with the UVES spectrograph installed at the VLT/UT2 Kueyen telescope (Paranal Observatory, ESO, Chile) during several campaigns (see Table 1) and have previously been used in the analysis of stellar parameters, together with the derivation of precise chemical abundances (see e.g. Santos et al. 2004; Sousa et al. 2011a,b; Delgado Mena et al. 2012).

High spectral resolving power $(R=80000)$ and a relatively high signal-to-noise ratio $(\mathrm{S} / \mathrm{N})$ are optimal for properly analysing the $\mathrm{NH}$ band at $3360 \AA$. The average $\mathrm{S} / \mathrm{N}$ of our sample in the studied regions is 150 .

The sample consists of 74 dwarf stars (42 stars with planets $^{1}$ and 32 comparison stars without detected planets) with effective temperatures between $4583 \mathrm{~K}$ and $6431 \mathrm{~K}$, metallicities from -0.45 to $0.55 \mathrm{dex}$, and surface gravities from 3.69 to 4.82 dex. Comparison sample stars were taken from HARPS and CORALIE samples.

\section{Analysis}

\subsection{Stellar parameters and chemical abundances}

The stellar parameters used in this study were taken from Sousa et al. (2008, 2011a,b), Tsantaki et al. (2013) and González Hernández et al. (2010, 2013). All stellar parameters used were derived by measuring equivalent widths of Fe I and Fe II lines using the code ARES (Sousa et al. 2007). Also, chemical abundances of elements other than $\mathrm{N}$ were adapted in targets with more recent stellar parameters (derived by our group with the same technique). To do this, the uncertainties presented in their original source were followed so systematic effects in the chemical abundances or the stellar parameters are negligible, not affecting the consistency of our results.

Chemical abundances of the elements with spectral lines present in the NH band were obtained from Adibekyan et al. (2012b) and González Hernández et al. (2010, 2013). These elements are $\mathrm{Ca}, \mathrm{Ti}, \mathrm{Mn}$, and $\mathrm{Si}$ (note that the abundances of these elements were simply scaled with iron in Ecuvillon et al. (2004)). The $\mathrm{N}$ abundance is also affected by $\mathrm{C}$ and $\mathrm{O}$ molecular equilibrium. The $\mathrm{C}$ and $\mathrm{O}$ abundances were obtained from Suárez-Andrés et al. (in prep.) and Bertran de Lis et al. (2015), respectively. However, there are 26 stars with no previous measurements of $\mathrm{O}$. We have decided to use the aforementioned results to interpolate for a given set of stellar parameters and use

\footnotetext{
1 Data from: www . exoplanet.eu
} 

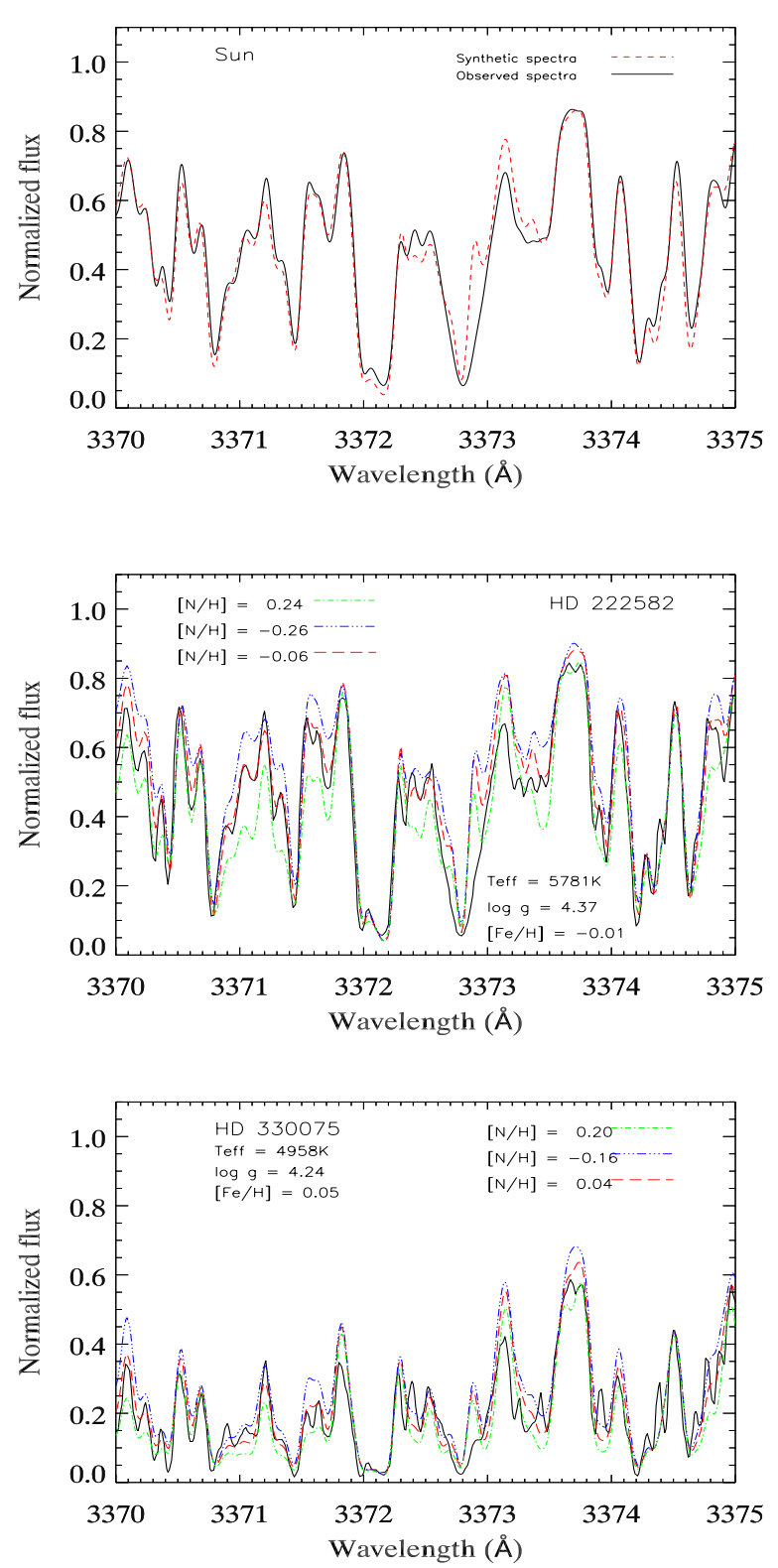

Fig. 1. Top panel: solar observed (solid line) and synthetic (dotted line) spectra in the spectral region $\lambda \lambda 3370-3375 \AA$. Middle and bottom panels: observed (solid) and synthetic spectra (green-dotted lined, red-dashed and blue-dashed dotted) of HD 222582 and HD 330075.

these new $\mathrm{O}$ abundances to calculate the molecular equilibrium. Our tests show that $\mathrm{N}$ abundances are unaffected by changes of the order of \pm 0.2 dex in $\mathrm{C}$ and $\mathrm{O}$.

Nitrogen abundances were determined using a standard local thermodynamic equilibrium (LTE) analysis with the spectral synthesis code MOOG (Sneden 1973, 2013 version) and a grid of Kurucz (1993) ATLAS9 atmospheres. All the atmospheric parameters, $T_{\text {eff }}, \log g, \xi_{t}$ and $[\mathrm{Fe} / \mathrm{H}]$ were taken, as already mentioned, from Sousa et al. (2008, 2011a,b), Tsantaki et al. (2013) and González Hernández et al. (2010, 2013). The adopted solar abundances for nitrogen and iron were $\log \epsilon(N)_{\odot}=8.05$ dex and $\log \epsilon(\mathrm{Fe})_{\odot}=7.47$ (Santos et al. 2004).

\subsection{The $\mathrm{NH}$ band}

The NH band is the strongest feature observed in the $\lambda \lambda 3345-3375 \AA$ spectral region. We determined $\mathrm{N}$ abundances by fitting synthetic spectra to the data in this wavelength range. The dissociation potential used for $\mathrm{NH}$ spectra is $D_{o}=3.37 \mathrm{eV}$, as recommended in Grevesse et al. (1990). The complete line list used in this study was obtained from Ecuvillon et al. (2004), which was calibrated with the KURUCZ ATLAS spectrum (Kurucz et al. 1984) using the abundance $\log \epsilon(N)_{\odot}=8.05$ dex.

The number and the strength of the atomic lines of different elements in the spectral region of the $\mathrm{NH}$ band increase with metallicity. The presence of many blended lines and numerous strong molecular bands make the placement of the continuum level very difficult. The high-resolution solar atlas (Kurucz et al. 1984) can help to achieve a reliable continuum placement for solar-type stars (e.g. Ecuvillon et al. 2004). However, large variations of metallicity and effective temperature among the stars in our sample do not allow us to use the solar spectrum as a reference. To account for this effect, we have generated a grid of synthetic spectra for all the stars in our sample. Synthetic spectra are calculated for a given set of stellar parameters and variations of nitrogen abundances $[\mathrm{N} / \mathrm{H}]$ between -0.7 and 0.5 . Our reference points for the continuum placement are those for which the flux variation (for a given $T_{\text {eff }}$ ) due to the $\mathrm{N}$ abundance changes from $[\mathrm{N} / \mathrm{Fe}]=-0.7$ to +0.5 , is less than $1 \%$. This strategy is demonstrated in Fig. 1 for two stars with different atmospheric parameters. Rotational broadening was set as a free parameter (it was fixed in Ecuvillon et al. 2004) with $v \sin i$ varying between 0.0 and 14.0 with a step of $1 \mathrm{~km} \mathrm{~s}^{-1}$. Macroturbulence was not taken into account. In order to find the best fit abundance value for each star, we used the FITTING program (González Hernández et al. 2011) and the MOOG synthesis code in its 2013 version. The best fit was obtained using a $\chi^{2}$ minimization procedure by comparing each synthetic spectrum with the observed one in the following spectral regions: $\lambda \lambda 3344.0-3344.3 \AA, \lambda \lambda 3346.2-3346.7 \AA$ $\lambda \lambda 3353.8-3354.4 \AA, \lambda \lambda 3357.4-3358.0 \AA, \lambda \lambda 3358.5-3359.7 \AA$, $\lambda \lambda 3360.3-3362.0 \AA, \lambda \lambda 3364.1-3364.8 \AA, \lambda \lambda 3370.8-3371.8 \AA$, $\lambda \lambda 3374.9-3375.4 \AA$. These spectral regions were chosen because of the presence of relatively strong NH features that allow reliable abundance measurements. We use a $\chi^{2}$ comparison for the observed and synthetic spectra, and we define $\chi^{2}=\sum_{i=1}^{N}\left(F_{i}-S_{i}\right)^{2} / N$, where $F_{i}$ and $S_{i}$ are the observed and synthetic fluxes respectively at wavelength point $i$. The $\chi^{2}$ for each spectral region was normalised with the number of points, $N$. Best-fit nitrogen abundances are extracted from each spectral range and the final nitrogen abundance for each star is then computed as the average of these values.

The FITTING program creates the following input data, required by MOOG: the atmospheric model, the line list, the range of nitrogen abundance of the grid of synthetic spectra, and the wavelength at which we want to analyse these abundances. To obtain the final abundance value, we analysed ten different ranges but use only those that have abundance values within $1 \sigma$.

In Fig. 1 we show the observed and synthetic spectra for the Sun and two stars that are depicted for different temperatures and metallicities within our sample. For these two stars, three different nitrogen abundances are also shown.

To examine how variations in the atmospheric parameters affect the $\mathrm{NH}$ abundances, we test $[\mathrm{N} / \mathrm{H}]$ sensitivity in stars with very different parameter values, given the wide range of stellar parameters. For each set of stars we tested the nitrogenabundance sensitivity to changes in the atmospheric parameter 


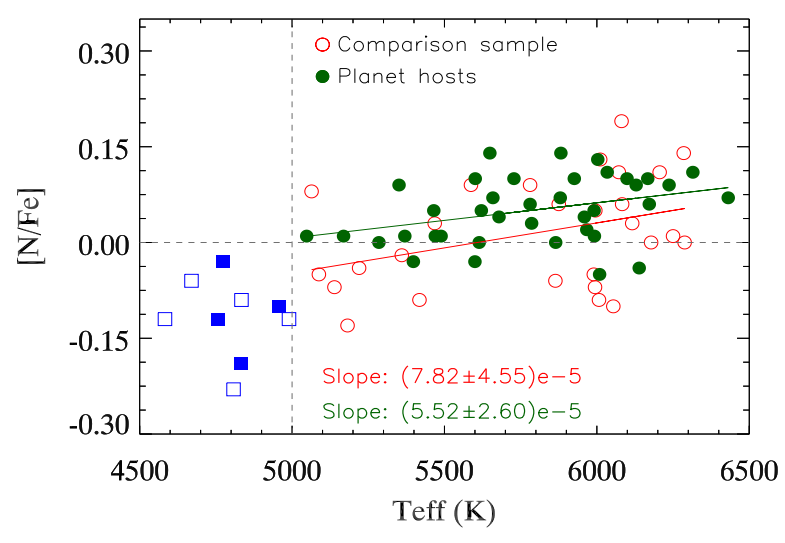

Fig. 2. $[\mathrm{N} / \mathrm{Fe}]$ abundance ratio of the stars in this study plotted against effective temperature, $T_{\text {eff }}$. Filled green circles represent planet hosts and open red circles the comparison sample. Filled blue squares represent cool planet hosts while open blue squares represent single stars. The vertical dashed line at $5000 \mathrm{~K}$ separates the cool stars from the studied sample.

( $\pm 100 \mathrm{~K}$ for $T_{\text {eff }}, \pm 0.2$ dex in $\log g, \pm 0.2$ in metallicity). The results are shown in Table 2. The effect of microturbulence was not taken into account because an increase of $0.3 \mathrm{~km} \mathrm{~s}^{-1}$ produced an average decrease of 0.002 dex in nitrogen abundance, which is negligible in comparison with the effects of other parameters. The error due to continuum placement of 0.1 dex was considered for all stars. All effects were added quadratically to obtain the final uncertainties in nitrogen abundances using the following relation:

$\Delta[\mathrm{N} / \mathrm{H}]=\left(\Delta_{\sigma}^{2}+\Delta_{T_{\mathrm{eff}}}^{2}+\Delta_{\log g}^{2}+\Delta_{\text {met }}^{2}+\Delta_{\text {cont }}^{2}\right)^{1 / 2}$.

\section{Results}

We analysed near-UV high-resolution spectra of 42 planet host stars and 32 comparison stars, as mentioned in Sect. 2. We aim to explore possible differences in nitrogen abundances between the two samples. Our results for planet-host are presented stars in Table A.1 and for the comparison sample in Table A.2.

In Fig. 2 we show the $[\mathrm{N} / \mathrm{Fe}]$ abundance ratio as a function of $T_{\text {eff }}$. Stars with effective temperatures below $5000 \mathrm{~K}$ were excluded from the analysis because of uncertainties in the behaviour of those cool stars: we find no explanation for the decrease in nitrogen abundance as we move to lower temperatures. The vertical dashed line at $5000 \mathrm{~K}$ separates the cool stars from the sample studied. From now on in this paper, all results and conclusions will refer to stars with $T_{\text {eff }}>5000 \mathrm{~K}$ (65 stars). Because of the high dispersion, we did not find any clear trend of [N/Fe] with $T_{\text {eff }}$. However, it can be seen that most of the planethost stars have $[\mathrm{N} / \mathrm{Fe}]>0$.

We have also looked for distinguishable trends between these samples by representing $[\mathrm{N} / \mathrm{Fe}]$ and $[\mathrm{N} / \mathrm{H}]$ abundance ratios as functions of $[\mathrm{Fe} / \mathrm{H}]$ for both samples (see Fig. 3). These plots indicate that both samples behave approximately similarly. However, there seems to be a steeper trend in $[\mathrm{N} / \mathrm{H}] \mathrm{vs}$. $[\mathrm{Fe} / \mathrm{H}]$ for stars with planets, whereas stars without planets almost maintain the 1:1 relation. We note the same behaviour in the bottom panel of Fig. $3([\mathrm{~N} / \mathrm{Fe}]$ vs. $[\mathrm{Fe} / \mathrm{H}])$, where the stars with planets below and above solar metallicity have values of $[\mathrm{N} / \mathrm{Fe}]$ lower and higher than zero respectively. Unfortunately, owing to the metal-rich nature of giant-planet hosts, the number of stars with giant planets at metallicities below solar is too small for us to be
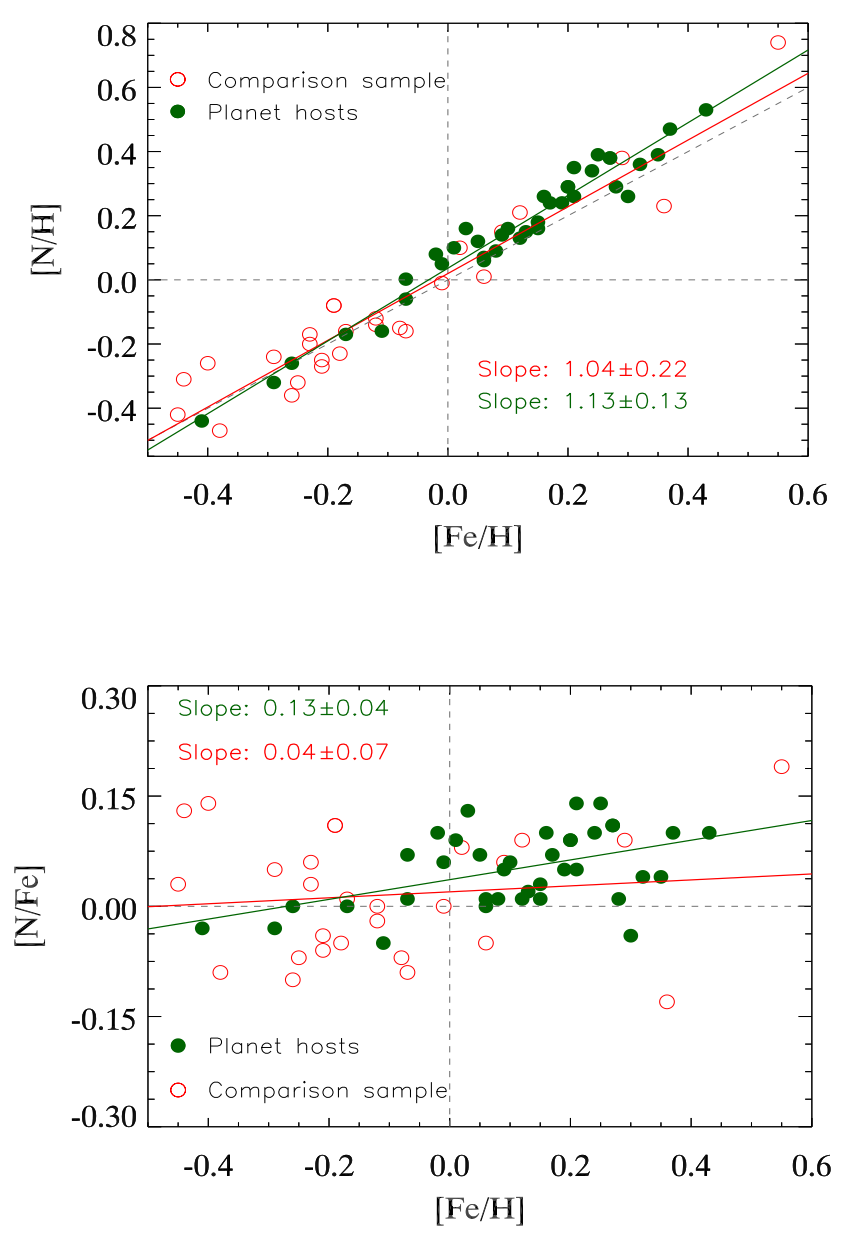

Fig. 3. $[\mathrm{N} / \mathrm{H}]$ and $[\mathrm{N} / \mathrm{Fe}]$ abundance ratios of our sample stars versus metallicity, $[\mathrm{Fe} / \mathrm{H}]$. Filled green circles represent planet hosts and open red circles, the comparison sample. Dashed lines stand for solar values.

able to confirm this behaviour. The observed trend may simply be related to Galactic chemical evolution.

In the top panel of Fig. 4 we show the $[\mathrm{N} / \mathrm{H}]$ abundance distributions for both samples. As can be seen, there is an offset between the samples, which is expected because planet-host stars are metal rich as compared with single stars. We expect this result because, if nitrogen scales with iron, then we can expect higher $[\mathrm{N} / \mathrm{H}]$ because giant-planet host stars are enhanced in $\mathrm{Fe}$. In the bottom panel of Fig. 4 we show the $[\mathrm{N} / \mathrm{Fe}]$ abundance distribution. We see that most of the stars with planets have $[\mathrm{N} / \mathrm{Fe}] \geq 0$ ( $\sim 90$ per cent $)$, as opposed to the single-star sample, more spread than the stars with planets sample. In this case, only $\sim 60$ per cent of the stars have $[\mathrm{N} / \mathrm{Fe}] \geq 0$. A KolmogorovSmirnov (K-S) test predicts the $\sim 0.06$ probability $\left(P_{\mathrm{KS}}\right)$ that stars with and without planets come from the same distribution. The distribution of abundance ratios $[\mathrm{N} / \mathrm{Fe}]$ vs. $[\mathrm{Fe} / \mathrm{H}]$ of stars without planets shows a weak linear increasing trend (see Fig. 3), although the slope is consistent with zero. The number of points may not be sufficient to really confirm this increasing trend. Thus, we may conclude that stars with planets are, on average, nitrogen rich when compared to single stars, but it may be due to the metal-rich nature of the planet host stars.

If we extend the study of nitrogen abundance to metal-poor stars (Israelian et al. 2004), we will ensure that the behaviour seen in Fig. 3 is part of the same trend observed in these metalpoor stars down to metallicites $\sim-2$ dex. In Fig. 5 we can see 

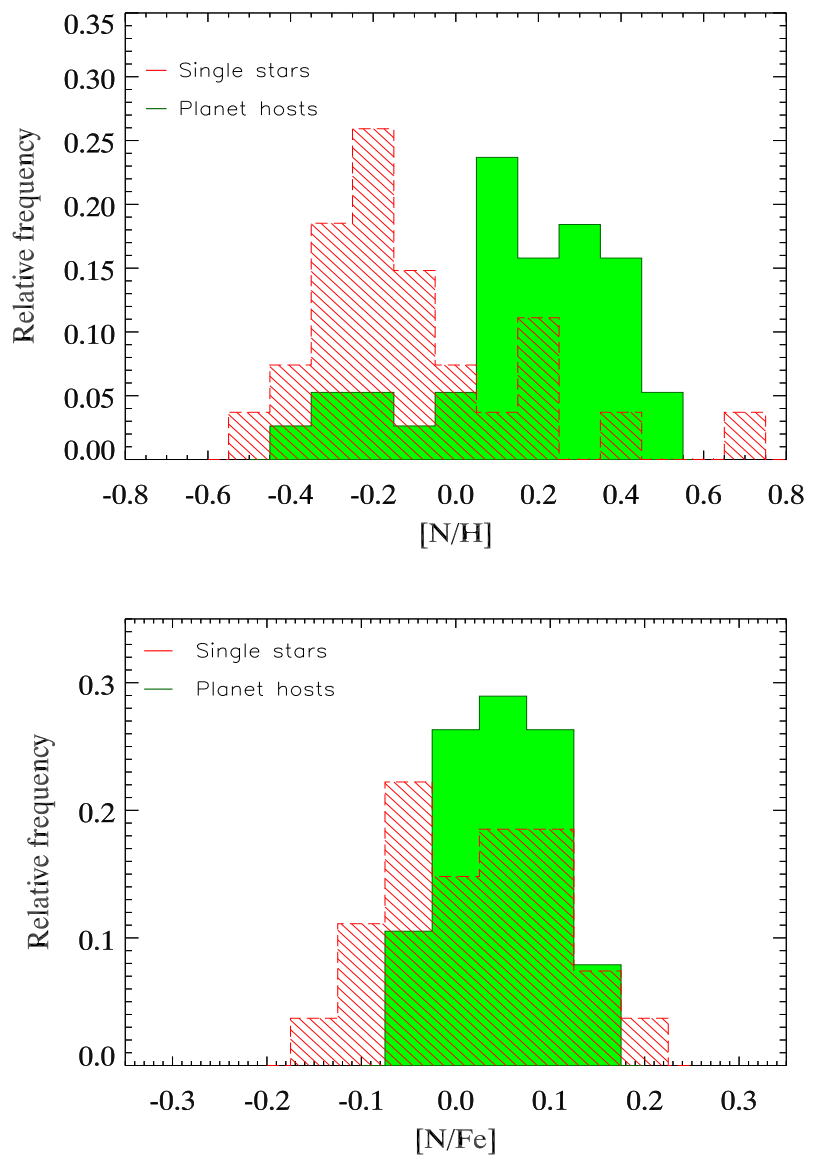

Fig. 4. $[\mathrm{N} / \mathrm{H}]$ and $[\mathrm{N} / \mathrm{Fe}]$ abundance distributions.

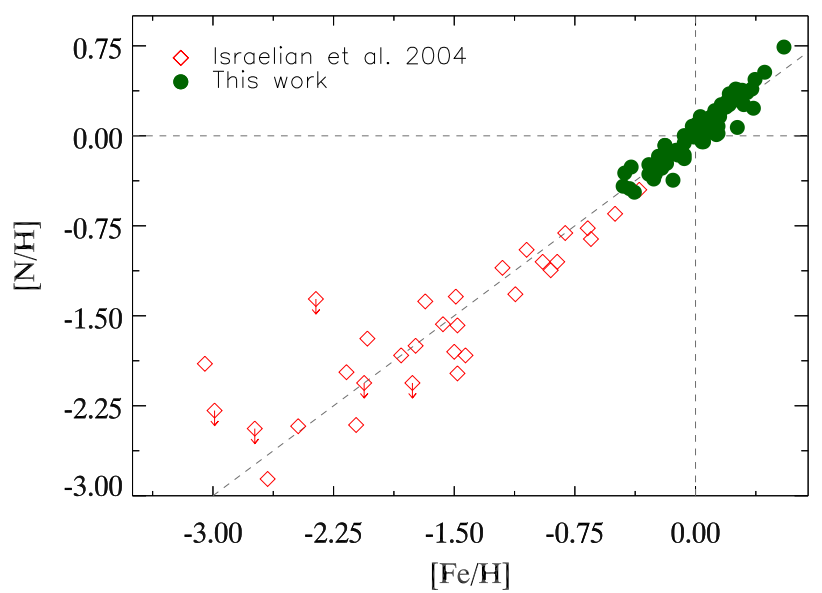

Fig. 5. $\mathrm{N} / \mathrm{H}$ plotted against $\mathrm{Fe} / \mathrm{H}$ for metal-poor stars from Israelian et al. (2004) indicated by red squares and stars from this study indicated by green circles.

how these two sets (Israelian et al. 2004, and this study) follow the same trend down to metallicities of -2.0 dex because nitrogen behaves like a secondary production element in this range of metallicities. At metallicities lower than -2.0 dex, we see a signature of primary $\mathrm{N}$.

\section{Galactic evolution of N: dependance on age}

Interpretation of chemical abundances in terms of stellar ages can be helpful to constrain the effects of Galactic chemical evolution.

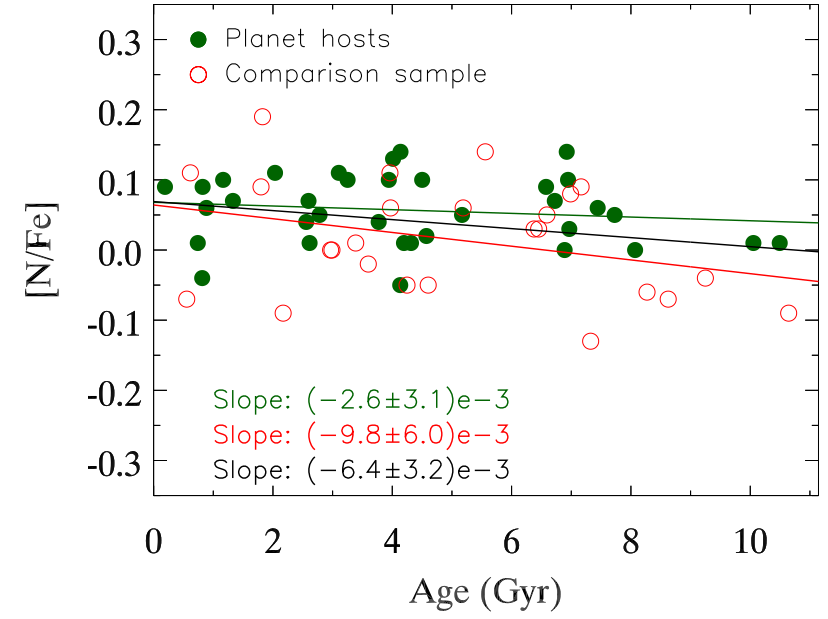

Fig. 6. Abundance ratio of $[\mathrm{N} / \mathrm{Fe}]$ as a function of stellar age for the stars belonging to the thin disk. Linear fit is provided for the whole sample (black line) and each sub-sample (red and green lines).

Stellar ages for our sample were estimated applying stellar evolutional models from the Padova group (Bressan et al. 2012), using the web interface ${ }^{2}$ (see Sousa et al. 2011a, for more details).

Nissen 2015 performed a detailed study of abundance ratios of several elements, such as $\mathrm{C}, \mathrm{O}$ and $\mathrm{Si}$, as a function of the stellar age. They conclude that each element behaves in a different way regarding the stellar age, suggesting that more variables such an evolving initial mass function and asymptotic giant branch stars should also be considered. Although that work is only for solar twin stars, we try to extend this result to solar type stars and for nitrogen, an element not studied in that work.

In the top panel of Fig. 6 we show $[\mathrm{N} / \mathrm{Fe}]$ abundances as a function of stellar age for the thin disk stars. There seem to be a weak trend in the behaviour of nitrogen, as its abundance decreases with age. Although single stars appear to show a steeper decreasing trend towards older stars than planet hosts stars, probably due to the low number of points and the high dispersion of the abundance measurements, the slopes of these two trends are consistent within their error bars.

\section{Kinematic properties}

To study the kinematic properties of the sample stars and which stellar populations they belong to, we applied both purely chemical (e.g. Adibekyan et al. 2011; Recio-Blanco et al. 2014) and kinematic approaches (e.g. Bensby et al. 2003; Reddy et al. 2006). The Galactic space velocity components of the stars were calculated as in Adibekyan et al. (2012b) using the astrometric ${ }^{3}$ and radial velocity data of the stars. The average errors in the $U$, $V$, and $W$ velocities are approximately $2-3 \mathrm{~km} \mathrm{~s}^{-1}$.

To assess the likelihood of the stars being members of different stellar populations we followed Reddy et al. (2006), and adopted the results of Bensby et al. (2003) for the population fractions. According to this separation, among the 65 stars, we have 58 ( 89 per cent) stars from the thin disc, four from the thick disc, and three transition stars that do not belong to any group.

The separation of the Galactic stellar components based only on stellar abundances is probably superior to that based

\footnotetext{
2 http://stev.oapd.inaf.it/cgi-bin/cmd

3 The SIMBAD Astronomical Database (http://simbad. u-strasbg.fr/simbad/) was used.
} 
Table 2. Sensitivity of the nitrogen abundance derived from the NH band at $3360 \AA$.

\begin{tabular}{cccc}
\hline \hline & \multicolumn{3}{c}{ Star } \\
& \multicolumn{3}{c}{$\left(T_{\text {eff }} ; \log g ;[\mathrm{Fe} / \mathrm{H}]\right)$} \\
\cline { 2 - 4 } & HD 93083 & HD 222582 & HD 39091 \\
$\Delta T_{\text {eff }}= \pm 100 \mathrm{~K}$ & $\pm 048 ; 4.32 ; 0.04)$ & $(5779 ; 4.32 ;-0.01)$ & $(6003 ; 4.42 ; 0.09)$ \\
\cline { 2 - 4 } & HD 11964A & HD 93083 & HD 1237 \\
& $(5332 ; 3.90 ; 0.10)$ & $(5105 ; 4.43 ; 0.09)$ & $(5514 ; 4.50 ; 0.07)$ \\
\cline { 2 - 4 }$\Delta \log g= \pm 0.2 \mathrm{dex}$ & $\mp 0.01$ & $\mp 0.05$ & $\mp 0.03$ \\
\hline & HD 4208 & HD 69830 & HD 73256 \\
\cline { 2 - 4 }$\Delta([\mathrm{Fe} / \mathrm{H}])= \pm 0.2 \mathrm{dex}$ & $\mp 0.16$ & $(5402 ; 4.40 ;-0.06)$ & $(5526 ; 4.42 ; 0.23)$ \\
\hline
\end{tabular}

Notes. Changes of $100 \mathrm{~K}$ in $T_{\text {eff }}, 0.2$ dex in gravity, and 0.2 in $[\mathrm{Fe} / \mathrm{H}]$ were applied.

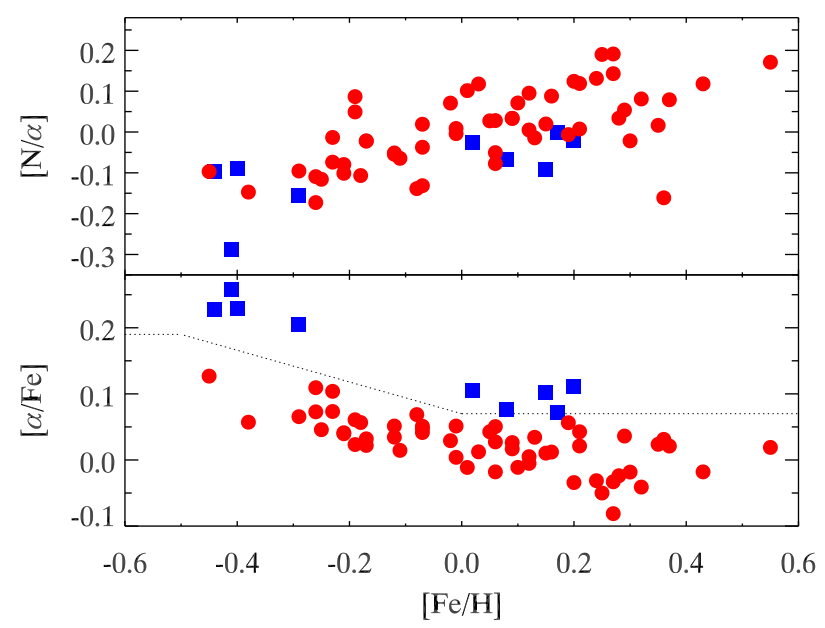

Fig. 7. $[\mathrm{N} / \alpha]$ plotted against $[\mathrm{Fe} / \mathrm{H}](t o p)$ and $[\alpha / \mathrm{Fe}]$ plotted against $[\mathrm{Fe} / \mathrm{H}]($ bottom $)$ for the sample. Stars that are enhanced in $\alpha$-elements are shown in blue squares and the thin-disc stars (non- $\alpha$-enhanced) are represented by red filled circles.

on kinematics alone (e.g. Navarro et al. 2011; Adibekyan et al. 2011) because chemistry is a relatively more stable property of sunlike stars than spatial positions and kinematics. We used the position of the stars in the $[\alpha / \mathrm{Fe}]-[\mathrm{Fe} / \mathrm{H}]$ plane (here $\alpha$ refers to the average abundance of $\mathrm{Si}$ and $\mathrm{Ti}$ ) to separate the thin- and thick-disc stellar components. We adopt the boundary (separation line) between the stellar populations from Adibekyan et al. (2011). According to our separation, 53 stars $(\approx 82$ per cent) are not enhanced in $\alpha$-elements and belong to the thin-disc population. The $[\alpha / \mathrm{Fe}]$ versus $[\mathrm{Fe} / \mathrm{H}]$ plot for the sample stars is shown in the bottom panel of Fig. 7 .

In the top plot of Fig. 7 we show the dependence of $[\mathrm{N} / \alpha]$ on the metallicity. The figure shows that the $[\mathrm{N} / \alpha]$ abundance ratio correlates with the metallicity. This trend is expected if the $\mathrm{N}$ abundance scales with the iron abundance, as was suggested above.

\section{The star-planet connection}

The increasing number of exoplanets discovered via different methods has led to a wide diversity of parameters (masses, radii, eccentricity, orbital period, etc.) being known for these planets. Many studies have suggested that the formation of giant

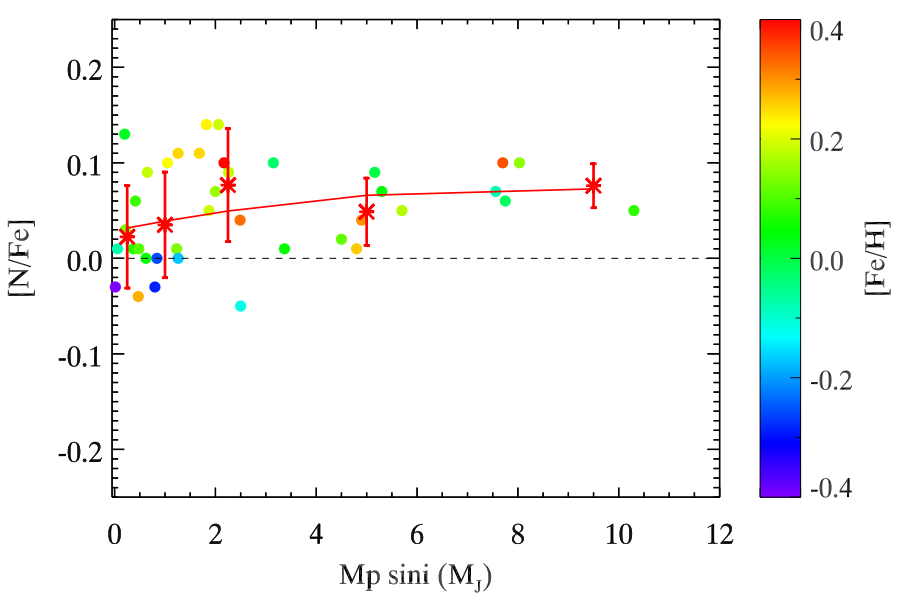

Fig. 8. $[\mathrm{N} / \mathrm{Fe}]$ plotted against $M_{p} \sin i$, with $[\mathrm{Fe} / \mathrm{H}]$ in the auxiliary axis. Dots represent the whole sample and red asterisks represent binned values. We also represent in red a second-degree polinomial fit for those binned values.

planets correlates with the metallicities of stellar hosts, (see Santos et al. 2001, 2004; Fischer \& Valenti 2005; Sousa et al. 2011a; Mortier et al. 2013). Moreover, it has been shown that the formation of planets (of both high and low masses) at low metallicities is favoured by enhanced $\alpha$-elements (Adibekyan et al. 2012a,c).

Models of planet formation require precise stellar abundances. Chemical abundances of planet hosts are useful when studying the properties of the planetary companion (Sousa et al. 2015). The relationship between $[\mathrm{N} / \mathrm{Fe}]$ and the mass of the planet is shown in Fig. 8. In those stars which host many planets, the most massive planet was considered in the plot. As we can see, the masses of the planets are between 0.015 and $10.3 M_{\mathrm{J}}$, but most stars have planets with less than $4 M_{\mathrm{J}}$ orbiting around them. To remove this bias, which is due to the lack of planets with higher masses, we created bins with increasing steps, with sizes of $0.5,1,1.5 M_{\mathrm{J}}$ and two bins of $4 M_{\mathrm{J}}$. Error bars indicate the standard deviation of each bin. Although the results are biased because of the lack of data in the highest-mass part, we cannot obtain a clear relationship between $[\mathrm{N} / \mathrm{Fe}]$ and the planetary mass (covariance $s_{x, y}=0.031$; where $x=M_{\mathrm{J}}$ and $y=[\mathrm{N} / \mathrm{Fe}]$ ). We see an increase of the $[\mathrm{N} / \mathrm{Fe}]$ ratio in the first mass bins followed by constant $[\mathrm{N} / \mathrm{Fe}]$ value for the more massive planets. 
Even so, the number of stars is not statistically significant to confirm this.

\section{Discussion and conclusions}

We present nitrogen abundances for 74 solar-type stars observed with the UVES spectrograph at the VLT/UT2 Kueyen telescope (Paranal Observatory, ESO, Chile). In our sample, 42 of the 74 are planet-hosts and 32 stars do not have any known planetary companion. All targets have been studied using spectral synthesis in the NH band at $3360 \AA$. The stars in our sample have effective temperatures between $4583 \mathrm{~K}$ and $6431 \mathrm{~K}$, metallicities from -0.45 to 0.55 dex, and surface gravities from 3.69 to 4.82 dex. We performed a detailed analysis of this sample to obtain precise nitrogen abundances and investigate possible differences between the stars with planets and the single stars. Nitrogen abundances of both samples behave in a similar way regarding $T_{\text {eff }}, \log g$, and $[\mathrm{Fe} / \mathrm{H}]$.

We extended our results to the metal-poor regime, comparing our results with previous work by Israelian et al. (2004). Both results can be accommodated under a common fit in an $[\mathrm{N} / \mathrm{H}]$ versus $[\mathrm{Fe} / \mathrm{H}]$ plot until metallicities down to -2.0 dex (where the production of nitrogen has a secondary origin), suggesting that both metal-poor stars and solar-like stars follow the same behaviour.

The correlation between the presence of planets and nitrogen abundances is expected: The large amount of nitrogen in a protoplanetary disc would favour the formation of massive giant planets through the core accretion scenario.

We searched for correlations between planet mass and [N/Fe] abundance ratio, but did not find any significant correlation.

Acknowledgements. J.I.G.H. acknowledges financial support from the Spanish Ministry of Economy and Competitiveness (MINECO) under the 2011 Severo Ochoa program MINECO SEV-2011-0187 and the 2013 Ramón y Cajal programme MINECO RYC-2013-14875, and the Spanish ministry project MINECO AYA2014-56359-P. E.D.M and V.Zh.A. acknowledge the support from the Fundação para a Ciência e Tecnologia, FCT (Portugal) in the form of the grants SFRH/BPD/76606/2011 and SFRH/BPD/70574/2010 respectively. V.Zh.A also aknowledges the support from COST Action TD1308 through STSM grant with reference Number: COST-STSM-TD 1308-32051. N.C.S. an S.G.S. also acknowledge support from FCT through Investigador FCT contracts of reference IF/00169/2012 and IF/00028/2014, and POPH/FSE (EC) by FEDER funding through the "Programa Operacional de Factores de Competitividade COMPETE". This work was supported by Fundação para a Ciência e a Tecnologia (FCT) through the research grant UID/DIS/04434/2013 (POCI-01-0145FEDER-007672) PTDC/FIS-AST/7073/2014 and PTDC/FIS-AST/1526/2014.

\section{References}

Adibekyan, V. Z., Santos, N. C., Sousa, S. G., Israelian, G. 2011, A\&A, 535, L11 Adibekyan, V. Z., Santos, N. C., Sousa, S. G., et al. 2012a, A\&A, 543, A89 Adibekyan, V. Z., Sousa, S. G., Santos, N. C., et al. 2012b, A\&A, 545, A32 Adibekyan, V. Z., Delgado Mena, E., Sousa, S. G., et al. 2012c, A\&A, 547, A36 Adibekyan, V. Z., González Hernández, J. I., Delgado Mena, E., et al. 2014, A\&A, 564, L15

Adibekyan, V., Santos, N. C., Figueira, P., et al. 2015, A\&A, 581, L2
Bensby T., Feltzing S., Lundström I. 2003, A\&A, 410, 527

Bertran de Lis, S., Delgado Mena, E., Adibekyan, V. Z., Santos, N. C., \& Sousa, S. G. 2015, A\&A, 576, A89

Bessell, M. S., \& Norris, J. 1982, ApJ, 263, L29

Bressan, A., Marigo, P., Girardi, L., et al. 2012, MNRAS, 427, 127

Buchhave, L. A., Latham, D. W., Johansen, A., et al. 2012, Nature, 486, 375

Carbon, D. F., Barbuy, B., Kraft, R. P., Friel, E. D., \& Suntzeff, N. B. 1987, PAGP, 99, 335

Chavero, C., de La Reza, R., Domingos, R. C., et al. 2010, A\&A, 517, A40

Da Silva, R., Milone, A. d. C., \& Rocha-Pinto, H. J. 2015, A\&A, 580, A24

Delgado Mena, E., Israelian, G., González Hernández, J. I., Santos, N. C., \& Rebolo, R. 2012, ApJ, 746, 47

Ecuvillon, A., Israelian, G., Santos, N. C., et al. 2004, A\&A, 418, 703

Fischer, D. A., \& Valenti, J. 2005, ApJ, 622, 1102

Ghezzi, L., Cunha, K., Smith, V. V., et al. 2009, ApJ, 698, 451

Gonzalez, G. 1997, MNRAS, 285, 403

González Hernández, J. I., Israelian, G., Santos, N. C., et al. 2010, ApJ, 720, 1592

González Hernández, J. I., Casares, J., Rebolo, R., et al. 2011, ApJ, 738, 95

González Hernández, J. I., Delgado-Mena, E., Sousa, S. G., et al. 2013, A\&A, 552, A6

Grevesse, N., Lambert, D. L., Sauval, A. J., et al. 1990, A\&A, 232, 225

Henry, R. B. C., Edmunds, M. G., Köppen, J. 2000, ApJ, 541, 660

Ida, S., \& Lin, D. N. C. 2004, ApJ, 604, 388

Israelian, G., Santos, N. C., Mayor, M., \& Rebolo, R. 2001, Nature, 411, 163

Israelian, G., Santos, N. C., Mayor, M., \& Rebolo, R. 2003, A\&A, 405, 753

Israelian, G., Ecuvillon, A., Rebolo, R., et al. 2004, A\&A, 421, 649

Kurucz, R. 1993, in ATLAS9 Stellar Atmosphere Programs and $2 \mathrm{~km} \mathrm{~s}^{-2}$ grid, Kurucz CD-ROM (Cambridge, Mass.: Smithsonian Astrophysical Observatory), 13

Kurucz, R. L., Furenlid, I., Brault, J., \& Testerman, L. 1984, National Solar Observatory Atlas (Sunspot, New Mexico: National Solar Observatory)

Liang, Y. C., Zhao, G., \& Shi, J. R. 2001, A\&A, 374, 936

Maeder, A. 2009, Physics, Formation and Evolution of Rotating Stars (Berlin Heidelberg: Springer), Astron. Astrophys. Lib.

Maeder, A., \& Meynet, G. 2000, ARA\&A, 38, 143

Mandell, A. M., Ge, J., \& Murray, N. 2004, AJ, 127, 1147

Marigo, P. 2001, A\&A, 370, 194

Mordasini, C., Alibert, Y., Klahr, H., \& Henning, T. 2012, A\&A, 547, A111

Mortier, A., Santos, N. C., Sousa, S. G., et al. 2013, A\&A, 551, A112

Navarro J. F., Abadi M. G., Venn K. A., Freeman K. C., \& Anguiano B. 2011, MNRAS, 412, 1203

Nissen, P. E. 2015, A\&A, 579, A52

Pagel, B. E. J., \& Edmunds, M. G. 1981, ARA\&A, 19, 77

Pettini, M., Ellison, S. L., Bergeron, J., \& Petitjean, P. 2002, A\&A, 391, 21

Reddy B. E., Lambert D. L., \& Allende Prieto C. 2006, MNRAS, 367, 1329

Recio-Blanco, A., de Laverny, P., Kordopatis, G., et al. 2014, A\&A, 567, A5

Santos, N. C., Israelian, G., \& Mayor, M. 2001, A\&A, 373, 1019

Santos, N. C., García López, R. J., Israelian, G., et al. 2002, A\&A, 386, 1028

Santos, N. C., Israelian, G., Mayor, M., Rebolo, R., \& Udry, S. 2003, A\&A, 398, 363

Santos, N. C., Israelian, G., \& Mayor, M. 2004, A\&A, 415, 1153

Santos, N. C., Israelian, G., Mayor, M., et al. 2005, A\&A, 437, 1127

Santos, N. C., Adibekyan, V., Mordasini, C., et al. 2015, A\&A, 580, L13

Sneden, C. A. 1973, Ph.D. Thesis, Texas University, Austin, USA

Sousa, S. G., Santos, N. C., Israelian, G., Mayor, M., \& Monteiro, M. J. P. F. G. 2007, A\&A, 469, 783

Sousa, S. G., Santos, N. C., Mayor, M., et al. 2008, A\&A, 487, 373

Sousa, S. G., Santos, N. C., Israelian, G., et al. 2011a, A\&A, 526, A99

Sousa, S. G., Santos, N. C., Israelian, G., Mayor, M., \& Udry, S. 2011b, A\&A, 533, A141

Sousa, S. G., Santos, N. C., Mortier, A., et al. 2015, A\&A, 576, A94

Théado, L. S., \& Vauclair, S. 2012, ApJ, 744, 2

Tsantaki, M., Sousa, S. G., Adibekyan, V. Z., et al. 2013, A\&A, 555, A150

Valenti, J. A., \& Fischer, D. A. 2005, ApJS, 159, 141

van den Hoek, L. B., \& Groenewegen, M. A. T. 1997, A\&AS, 123, 305 


\section{Appendix A: Nitrogen abundances for stars}

\section{with and without planets}

Table A.1. Nitrogen abundances for a set of stars with planets.

\begin{tabular}{|c|c|c|c|c|c|}
\hline Star & $T_{\text {eff }}(\mathrm{K})$ & $\log g\left(\mathrm{~cm} \mathrm{~s}^{-2}\right)$ & $\xi_{t}\left(\mathrm{~km} \mathrm{~s}^{-1}\right)$ & {$[\mathrm{Fe} / \mathrm{H}]$} & {$[\mathrm{N} / \mathrm{H}]$} \\
\hline HD 142 & 6431 & 4.82 & 2.1 & 0.05 & $0.12 \pm 0.10$ \\
\hline HD 1237 & 5489 & 4.46 & 1.04 & 0.06 & $0.07 \pm 0.10$ \\
\hline HD 2039 & 5990 & 4.56 & 1.24 & 0.34 & $0.36 \pm 0.10$ \\
\hline HD 2638 & 5169 & 4.41 & 0.66 & 0.12 & $0.13 \pm 0.11$ \\
\hline HD 4203 & 5728 & 4.23 & 1.18 & 0.43 & $0.53 \pm 0.11$ \\
\hline HD 4208 & 5600 & 4.41 & 0.88 & -0.29 & $-0.32 \pm 0.12$ \\
\hline HD 16141 & 5786 & 4.17 & 1.1 & 0.15 & $0.18 \pm 0.10$ \\
\hline HD 17051 & 6237 & 4.46 & 1.31 & 0.2 & $0.29 \pm 0.13$ \\
\hline HD 19994 & 6315 & 4.44 & 1.66 & 0.27 & $0.38 \pm 0.12$ \\
\hline HD 20794 & 5398 & 4.41 & 0.7 & -0.41 & $-0.44 \pm 0.11$ \\
\hline HD 23079 & 6009 & 4.5 & 1.2 & -0.11 & $-0.16 \pm 0.10$ \\
\hline HD 27894 & 4833 & 4.3 & 0.33 & 0.26 & $0.07 \pm 0.13$ \\
\hline HD 28185 & 5621 & 4.36 & 0.92 & 0.19 & $0.24 \pm 0.12$ \\
\hline HD 30177 & 5601 & 4.34 & 0.89 & 0.37 & $0.47 \pm 0.14$ \\
\hline HD 39091 & 5991 & 4.4 & 1.09 & 0.09 & $0.14 \pm 0.10$ \\
\hline HD 50554 & 6129 & 4.41 & 1.11 & 0.01 & $0.10 \pm 0.12$ \\
\hline HD 52265 & 6167 & 4.44 & 1.28 & 0.24 & $0.34 \pm 0.12$ \\
\hline HD 65216 & 5614 & 4.46 & 0.81 & -0.17 & $-0.17 \pm 0.10$ \\
\hline HD 63454 & 4756 & 4.32 & 0.31 & 0.13 & $0.01 \pm 0.11$ \\
\hline HD 69830 & 5370 & 4.38 & 0.67 & -0.07 & $-0.06 \pm 0.10$ \\
\hline HD 70642 & 5659 & 4.43 & 0.81 & 0.17 & $0.24 \pm 0.11$ \\
\hline HD 72659 & 5926 & 4.24 & 1.13 & -0.02 & $0.08 \pm 0.10$ \\
\hline HD 73256 & 5465 & 4.36 & 1.0 & 0.21 & $0.26 \pm 0.10$ \\
\hline HD 74156 & 6099 & 4.34 & 1.38 & 0.16 & $0.26 \pm 0.11$ \\
\hline HD 75289 & 6139 & 4.35 & 1.22 & 0.3 & $0.26 \pm 0.12$ \\
\hline HD 82943 & 5992 & 4.42 & 1.06 & 0.28 & $0.29 \pm 0.13$ \\
\hline HD 93083 & 5048 & 4.32 & 0.81 & 0.08 & $0.09 \pm 0.10$ \\
\hline HD 106252 & 5880 & 4.4 & 1.13 & -0.07 & $0.00 \pm-0.10$ \\
\hline HD 114386 & 4774 & 4.37 & 0.01 & -0.09 & $-0.12 \pm 0.10$ \\
\hline HD 114729 & 5865 & 4.2 & 1.29 & -0.26 & $-0.26 \pm 0.10$ \\
\hline HD 117207 & 5649 & 4.31 & 0.95 & 0.21 & $0.35 \pm 0.10$ \\
\hline HD 117618 & 6003 & 4.45 & 1.16 & 0.03 & $0.16 \pm 0.10$ \\
\hline HD 11964A & 5285 & 3.81 & 0.95 & 0.06 & $0.06 \pm 0.11$ \\
\hline HD 208487 & 6172 & 4.54 & 1.22 & 0.1 & $0.16 \pm 0.11$ \\
\hline HD 210277 & 5470 & 4.26 & 0.9 & 0.15 & $0.16 \pm 0.10$ \\
\hline HD 213240 & 5967 & 4.28 & 1.22 & 0.13 & $0.15 \pm 0.10$ \\
\hline HD 216435 & 6034 & 4.21 & 1.27 & 0.27 & $0.38 \pm 0.12$ \\
\hline HD 216437 & 5882 & 4.25 & 1.25 & 0.25 & $0.39 \pm 0.11$ \\
\hline HD 216770 & 5351 & 4.31 & 0.85 & 0.2 & $0.29 \pm 0.10$ \\
\hline HD 217107 & 5679 & 4.32 & 1.15 & 0.35 & $0.39 \pm 0.10$ \\
\hline HD 222582 & 5781 & 4.37 & 1.02 & -0.01 & $0.05 \pm 0.10$ \\
\hline HD 330075 & 4958 & 4.24 & 0.32 & 0.05 & $-0.05 \pm 0.10$ \\
\hline
\end{tabular}


Table A.2. Nitrogen abundances for a set of stars without planets (comparison sample).

\begin{tabular}{|c|c|c|c|c|c|}
\hline Star & $T_{\text {eff }}(\mathrm{K})$ & $\log g\left(\mathrm{~cm} \mathrm{~s}^{-2}\right)$ & $\xi_{t}\left(\mathrm{~km} \mathrm{~s}^{-1}\right)$ & {$[\mathrm{Fe} / \mathrm{H}]$} & {$[\mathrm{N} / \mathrm{H}]$} \\
\hline HD 870 & 5360 & 4.4 & 0.79 & -0.12 & $-0.14 \pm 0.10$ \\
\hline HD 1581 & 5990 & 4.49 & 1.24 & -0.18 & $-0.23 \pm 0.10$ \\
\hline HD 3823 & 6054 & 4.37 & 1.44 & -0.26 & $-0.36 \pm 0.12$ \\
\hline HD 8326 & 4834 & 4.35 & 0.44 & 0.04 & $-0.05 \pm 0.10$ \\
\hline HD 8389A & 5182 & 4.33 & 0.81 & 0.36 & $0.23 \pm 0.11$ \\
\hline HD 9796 & 5139 & 4.34 & 0.49 & -0.25 & $-0.32 \pm 0.10$ \\
\hline HD 15337 & 5088 & 4.36 & 0.51 & 0.06 & $0.01 \pm 0.10$ \\
\hline HD 16270 & 4583 & 4.23 & 0.16 & 0.14 & $0.02 \pm 0.11$ \\
\hline HD 20807 & 5875 & 4.5 & 1.15 & -0.23 & $-0.17 \pm 0.10$ \\
\hline HD 21019 & 5468 & 3.93 & 1.1 & -0.45 & $-0.42 \pm 0.12$ \\
\hline HD 33636 & 5994 & 4.71 & 1.79 & -0.08 & $-0.15 \pm 0.11$ \\
\hline HD 35854 & 4808 & 4.35 & 0.16 & -0.14 & $-0.37 \pm 0.11$ \\
\hline HD 37226 & 6178 & 4.16 & 1.61 & -0.12 & $-0.12 \pm 0.10$ \\
\hline HD 40105 & 5064 & 3.69 & 0.91 & 0.02 & $0.10 \pm 0.10$ \\
\hline HD 44573 & 4990 & 4.42 & 0.61 & -0.07 & $-0.19 \pm 0.11$ \\
\hline HD 65907A & 5995 & 4.62 & 1.18 & -0.29 & $-0.24 \pm 0.10$ \\
\hline HD 72769 & 5587 & 4.3 & 0.86 & 0.29 & $0.38 \pm 0.11$ \\
\hline HD 73121 & 6083 & 4.27 & 1.33 & 0.09 & $0.15 \pm 0.11$ \\
\hline HD 76151 & 5781 & 4.44 & 0.93 & 0.12 & $0.21 \pm 0.10$ \\
\hline HD 103891 & 6072 & 4.05 & 1.5 & -0.19 & $-0.08 \pm 0.11$ \\
\hline HD 108063 & 6081 & 4.11 & 1.54 & 0.55 & $0.74 \pm 0.15$ \\
\hline HD 119629 & 6250 & 4.17 & 1.73 & -0.17 & $-0.16 \pm 0.11$ \\
\hline HD 141597 & 6285 & 4.38 & 1.23 & -0.4 & $-0.26 \pm 0.14$ \\
\hline HD 191033 & 6206 & 4.47 & 1.35 & -0.19 & $-0.08 \pm 0.13$ \\
\hline HD 205536 & 5418 & 4.36 & 0.79 & -0.07 & $-0.16 \pm 0.11$ \\
\hline HD 208068 & 6007 & 4.64 & 1.17 & -0.38 & $-0.47 \pm 0.13$ \\
\hline HD 211415 & 5864 & 4.42 & 1.01 & -0.21 & $-0.27 \pm 0.12$ \\
\hline HD 213042 & 4670 & 4.22 & 0.35 & 0.14 & $0.08 \pm 0.11$ \\
\hline HD 214094 & 6288 & 4.28 & 1.46 & -0.01 & $-0.01 \pm 0.10$ \\
\hline HD 220367 & 6116 & 4.45 & 1.44 & -0.23 & $-0.20 \pm 0.11$ \\
\hline HD 222335 & 5220 & 4.48 & 0.62 & -0.21 & $-0.25 \pm 0.10$ \\
\hline CD-436810 & 6011 & 4.41 & 1.09 & -0.44 & $-0.31 \pm 0.11$ \\
\hline
\end{tabular}

